Kragujevac Journal of Mathematics

Volume 44(3) (2020), Pages 459-473.

\title{
NECESSARY AND SUFFICIENT CONDITION FOR OSCILLATORY AND ASYMPTOTIC BEHAVIOUR OF SECOND-ORDER FUNCTIONAL DIFFERENTIAL EQUATIONS
}

\author{
SHYAM SUNDAR SANTRA ${ }^{1,2}$
}

\begin{abstract}
In this paper, necessary and sufficient conditions are obtained for oscillatory and asymptotic behaviour of solutions of second-order neutral delay differential equations of the form$$
\frac{d}{d t}\left[r(t) \frac{d}{d t}[x(t)+p(t) x(\tau(t))]\right]+q(t) G(x(\sigma(t)))=0, \quad \text { for } t \geq t_{0},
$$

under the assumption $\int^{\infty} \frac{1}{r(\eta)} d \eta=\infty$ for various ranges of the bounded neutral coefficient $p$. Our main tools are Lebesgue's dominated convergence theorem and Banach's contraction mapping principle. Further, an illustrative example showing the applicability of the new results is included.
\end{abstract}

\section{INTRODUCTION}

Consider a class of nonlinear neutral delay differential equations of the form:

$$
\frac{d}{d t}\left[r(t) \frac{d}{d t}[x(t)+p(t) x(\tau(t))]\right]+q(t) G(x(\sigma(t)))=0
$$

where

(A1) $r, q, \tau, \sigma \in C\left(\mathbb{R}_{+}, \mathbb{R}_{+}\right), p \in C\left(\mathbb{R}_{+}, \mathbb{R}\right)$ such that $\tau(t) \leq t, \sigma(t) \leq t$ for $t \geq t_{0}$, $\tau(t) \rightarrow \infty, \sigma(t) \rightarrow \infty$ as $t \rightarrow \infty$, with invertible $\tau$ when necessary;

$(A 2) G \in C(\mathbb{R}, \mathbb{R})$ is nondecreasing with satisfying the property $u G(u)>0$ for $u \neq 0$ and

(A3) $R(t)=\int_{0}^{t} \frac{d \eta}{r(\eta)} \rightarrow+\infty$ as $t \rightarrow \infty$.

Key words and phrases. Oscillation, nonoscillation, neutral, delay, nonlinear, Lebesgue's dominated convergence theorem, Banach's contraction mapping principle.

2010 Mathematics Subject Classification. Primary: 34C10. Secondary: 34C15, 35K40.

DOI 10.46793/KgJMat2003.459S

Received: November 08, 2017.

Accepted: June 06, 2018. 
Baculikova et al. [3] have studied the linear counterpart of (1.1),

$$
\frac{d}{d t}\left[r(t) \frac{d}{d t}[x(t)+p(t) x(\tau(t))]\right]+q(t) x(\sigma(t))=0,
$$

when $0 \leq p(t) \leq p_{0}<\infty$ and $(A 3)$ holds. The authors have obtained sufficient conditions for oscillation of solutions of (1.2) through some comparison results, where the comparison results are unpredictable. In [6], Džurina have studied (1.2) when $0 \leq p(t) \leq p_{0}<\infty$ and $(A 3)$ holds true. He has established sufficient condition for oscillation of solutions of (1.2) by comparison techniques. In [16], under various ranges of $p$, Santra studied oscillatory behaviour of the solutions of the following neutral differential equations

$$
\frac{d}{d t}[x(t)+p(t) x(t-\tau)]+q(t) G(x(t-\sigma))=0
$$

and

$$
\frac{d}{d t}[x(t)+p(t) x(t-\tau)]+q(t) G(x(t-\sigma))=f(t) .
$$

Also, sufficient conditions are obtained for existence of bounded positive solutions of (1.3). Tripathy et al. [18] have studied and obtained the sufficient conditions for oscillation, nonoscillation and asymptotic behavior of solutions of (1.1) provided $G$ could be linear or nonlinear. The motivation of the present work come from the above studies. Hence, in this work, an attempt is made to study the more general form of (1.2) without making any comparison. It seems that this method is the next alternative to the works $[3,6]$ when $p$ is bounded.

The neutral differential equations find numerous applications in natural sciences and technology. For instance, they are frequently used for the study of distributed networks containing lossless transmission lines (see, for e.g., [8]). In this paper, we restrict our attention to study (1.1), which includes a class of nonlinear functional differential equations of neutral type. In this direction we refer the reader to some of the works (see $[1,4,5,10,13,19,20]$ ) and the references cited therein.

By a solution to equation (1.1), we mean a function $x \in \mathrm{C}\left(\left[T_{x}, \infty\right), \mathbb{R}\right)$, where $T_{x} \geq t_{0}$, such that $r z^{\prime} \in \mathrm{C}^{1}\left(\left[T_{x}, \infty\right), \mathbb{R}\right)$, where

$$
z(t):=x(t)+p(t) x(\tau(t)), \quad \text { for } t \geq T_{x},
$$

and satisfies (1.1) on the interval $\left[T_{x}, \infty\right)$. A solution $x$ of $(1.1)$ is said to be proper if $x$ is not identically zero eventually, i.e., $\sup \{|x(t)|: t \geq T\}>0$ for all $T \geq T_{x}$. We assume that (1.1) possesses such solutions. A solution of (1.1) is called oscillatory if it has arbitrarily large zeros on $\left[T_{x}, \infty\right)$; otherwise, it is said to be nonoscillatory. (1.1) itself is said to be oscillatory if all of its solutions are oscillatory.

Remark 1.1. When the domain is not specified explicitly, all functional inequalities considered in this paper are assumed to hold eventually, i.e., they are satisfied for all $t$ large enough. 


\section{MAin Results}

In this section, necessary and sufficient conditions are obtained for oscillatory and asymptotic behaviour of solutions of second order nonlinear neutral differential equations of the form (1.1).

Lemma 2.1. Assume that (A1)-(A3) hold. If $x$ is an eventually positive solution of (1.1) such that the companion function z defined by (1.4) is also eventually positive, then $z$ satisfies

$$
z^{\prime}(t)>0 \text { and }\left(r z^{\prime}\right)^{\prime}(t)<0, \text { for all large } t .
$$

Proof. Suppose that $x(t)>0$ and $z(t)>0$ for $t \geq t_{1}$, where $t \geq t_{0}$. By $(A 1)$, we may assume without loss of generality that $x(\sigma(t))>0$ for $t \geq t_{1}$. From (1.1) and (A2), it follows that

$$
\left(r z^{\prime}\right)^{\prime}(t)=-q(t) G(x(\sigma(t)))<0, \quad \text { for } t \geq t_{1} .
$$

Consequently, $r z^{\prime}$ is nonincreasing on $\left[t_{1}, \infty\right)$ and thus either $z^{\prime}(t)<0$ or $z^{\prime}(t)>0$ for $t \geq t_{2}$, where $t_{2} \geq t_{1}$. If $z^{\prime}(t)<0$, then there exists $\varepsilon>0$ such that $r(t) z^{\prime}(t) \leq-\varepsilon$ for $t \geq t_{2}$, which yields upon integration over $\left[t_{2}, t\right) \subset\left[t_{2}, \infty\right)$ after dividing through by $r$ that

$$
z(t) \leq z\left(t_{2}\right)-\varepsilon \int_{t_{2}}^{t} \frac{1}{r(\eta)} d \eta, \quad \text { for } t \geq t_{2} .
$$

In view of (A3), letting $t \rightarrow \infty$ in (2.3) yields $z(t) \rightarrow-\infty$, which is a contradiction. Therefore, $z^{\prime}(t)>0$ for $t \geq t_{2}$. This completes the proof.

Remark 2.1. It follows from Lemma 2.1 that $\lim _{t \rightarrow \infty} z(t)>0$, i.e., there exists $\varepsilon>0$ such that $z(t) \geq \varepsilon$ for all large $t$.

Lemma 2.2. Assume that (A1)-(A3) hold. If $x$ is an eventually positive solution of (1.1) such that the companion function $z$ defined by (1.4) is bounded, then $z$ satisfies (2.1) for all large $t$.

Theorem 2.1. Assume that (A1)-(A3) hold and $-1<-a \leq p(t) \leq 0, a \geq 0$ for $t \in \mathbb{R}_{+}$. Furthermore, assume that

$(A 4) G$ is strictly sublinear, that is, $\frac{G(u)}{u^{\beta}} \geq \frac{G(v)}{v^{\beta}}, 0<u \leq v, \beta<1$, holds. Then every unbounded solution of (1.1) oscillates if and only if

$(A 5) \int_{T}^{\infty} q(\eta) G(\varepsilon R(\sigma(\eta))) d \eta=+\infty, T>0$ for every $\varepsilon>0$.

Proof. Suppose the contrary that $x$ is a nonoscillatory solution of (1.1). Then, there exists $t_{1} \geq t_{0}$ such that either $x(t)>0$ or $x(t)<0$ for $t \geq t_{1}$. Assume that $x(t)>0$, $x(\tau(t))>0$ and $x(\sigma(t))>0$ for $t \geq t_{1}$. Proceeding as in the proof of Lemma 2.1, we see $r z^{\prime}$ is nonincreasing and $z$ is monotonic on $\left[t_{2}, \infty\right)$, where $t_{2} \geq t_{1}$. We have the following two possible cases. 
Case 1. Let $z(t)<0$ for $t \geq t_{2}$. As $x$ is unbounded, there exists $T \geq t_{2}$ such that $x(T)=\max \left\{x(\eta): t_{2} \leq \eta \leq T\right\}$. Then, from (1.4), we have $x(T) \leq z(T)+x(\tau(T))<$ $x(T)$, which is a contradiction.

Case 2. Let $z(t)>0$ for $t \geq t_{2}$. By Lemma 2.1, (2.1) holds for $t \geq t_{3}$. Note that $\lim _{t \rightarrow \infty} r(t) z^{\prime}(t)$ exists. Upon using $z(t) \leq x(t)$ in $(2.2)$ and then integrating the final inequality from $t$ to $+\infty$, we obtain

$$
\int_{t}^{\infty} q(\eta) G(z(\sigma(\eta))) d \eta \leq r(t) z^{\prime}(t)
$$

that is,

$$
z^{\prime}(t) \geq \frac{1}{r(t)} \int_{t}^{\infty} q(\eta) G(z(\sigma(\eta))) d \eta
$$

for $t \geq t_{3}$. Let $t_{4}>t_{3}$ be a point such that

$$
R(t)-R\left(t_{4}\right) \geq \frac{1}{2} R(t), \quad t \geq t_{4}
$$

Then integrating (2.4) from $t_{4}$ to $t\left(>t_{4}\right)$, we get

$$
\begin{aligned}
z(t)-z\left(t_{4}\right) & \geq \int_{t_{4}}^{t} \frac{1}{r(\eta)} \int_{\eta}^{\infty} q(\zeta) G(z(\sigma(\zeta))) d \zeta d \eta \\
& \geq \int_{t_{4}}^{t} \frac{1}{r(\eta)} \int_{t}^{\infty} q(\zeta) G(z(\sigma(\zeta))) d \zeta d \eta
\end{aligned}
$$

that is,

$$
\begin{aligned}
z(t) & \geq\left(R(t)-R\left(t_{4}\right)\right) \int_{t}^{\infty} q(\zeta) G(z(\sigma(\zeta))) d \zeta \\
& \geq \frac{1}{2} R(t) \int_{t}^{\infty} q(\zeta) G(z(\sigma(\zeta))) d \zeta, \quad t \geq t_{4} .
\end{aligned}
$$

Using the fact that $r(t) z^{\prime}(t)$ is nonincreasing on $\left[t_{4}, \infty\right)$, we can find a constant $\varepsilon>0$ and $t_{5}>t_{4}$ such that $r(t) z^{\prime}(t) \leq \varepsilon$ for $t \geq t_{5}$ and hence $z(t) \leq \varepsilon R(t), t \geq t_{5}$. On the otherhand, (A3) implies that

$$
\begin{aligned}
G(z(\sigma(\zeta))) & =\frac{G(z(\sigma(\zeta)))}{z^{\beta}(\sigma(\zeta))} z^{\beta}(\sigma(\zeta)) \\
& \geq \frac{G(\varepsilon R(\sigma(\zeta)))}{\varepsilon^{\beta} R^{\beta}(\sigma(\zeta))} z^{\beta}(\sigma(\zeta)) .
\end{aligned}
$$

Consequently, (2.5) becomes

$$
z(t) \geq \frac{R(t)}{2} \int_{t}^{\infty} \frac{q(\zeta) G(\varepsilon R(\sigma(\zeta))) z^{\beta}(\sigma(\zeta))}{\varepsilon^{\beta} R^{\beta}(\sigma(\zeta))} d \zeta
$$


for $t \geq t_{5}$. If we define

$$
w(t)=\frac{1}{2} \int_{t}^{\infty} \frac{q(\zeta) G(\varepsilon R(\sigma(\zeta))) z^{\beta}(\sigma(\zeta))}{\varepsilon^{\beta} R^{\beta}(\sigma(\zeta))} d \zeta
$$

then $z(t) \geq R(t) w(t)$ for $t \geq t_{5}$. Now,

$$
\begin{aligned}
w^{\prime}(t) & \leq-\frac{1}{2} \frac{q(t) G(\varepsilon R(\sigma(t))) z^{\beta}(\sigma(t))}{\varepsilon^{\beta} R^{\beta}(\sigma(t))} \\
& \leq-\frac{1}{2} \frac{q(t) G(\varepsilon R(\sigma(t)))}{\varepsilon^{\beta}} w^{\beta}(\sigma(t)) \leq 0, \quad t \geq t_{5},
\end{aligned}
$$

implies that $w(t)$ is nonincreasing on $\left[t_{5}, \infty\right)$ and $\lim _{t \rightarrow \infty} w(t)$ exists. It is easy to verify that

$$
\begin{aligned}
{\left[w^{1-\beta}(t)\right]^{\prime} } & \leq-\frac{(1-\beta)}{2} w^{-\beta}(t) \frac{q(t) G(\varepsilon R(\sigma(t)))}{\varepsilon^{\beta}} w^{\beta}(\sigma(t)) \\
& \leq-\frac{(1-\beta)}{2} w^{-\beta}(t) \frac{q(t) G(\varepsilon R(\sigma(t)))}{\varepsilon^{\beta}} w^{\beta}(t) \\
& \leq-\frac{(1-\beta)}{2 \varepsilon^{\beta}} q(t) G(\varepsilon R(\sigma(t))),
\end{aligned}
$$

for $t \geq t_{5}$. Integrating (2.6) from $t_{5}$ to $t\left(>t_{5}\right)$, we obtain

$$
\frac{(1-\beta)}{2 \varepsilon^{\beta}} \int_{t^{5}}^{t} q(\eta) G(\varepsilon R(\sigma(\eta))) d \eta \leq-\left[w^{1-\beta}(\eta)\right]_{t_{5}}^{t}<w^{1-\beta}\left(t_{5}\right)<\infty,
$$

a contradiction to $(A 5)$.

If $x(t)<0$ for $t \geq t_{1}$, then we set $y(t):=-x(t)$ for $t \geq t_{1}$ in (1.1). Using (A2), we find

$$
\frac{d}{d t}\left[r(t) \frac{d}{d t}[y(t)+p(t) y(\tau(t))]\right]+q(t) H(y(\sigma(t)))=0, \quad \text { for } t \geq t_{1},
$$

where $H(u):=-G(-u)$ for $u \in \mathbb{R}$. Clearly, $H$ also satisfies $(A 2)$. Then, proceeding as above, we find the same contradiction.

Next, we suppose that $(A 5)$ does not hold. For $\varepsilon>0$, let us assume that

$$
\int_{T}^{\infty} q(\eta) G(\varepsilon R(\sigma(\eta))) d \eta \leq \frac{\varepsilon}{3}
$$

Consider

$$
\begin{aligned}
& M=\left\{x: x \in C\left(\left[t_{0}, \infty\right), \mathbb{R}\right), x(t)=0 \text { for } t \in\left[t_{0}, T\right]\right. \text { and } \\
&\left.\frac{\varepsilon}{3}[R(t)-R(T)] \leq x(t) \leq \varepsilon[R(t)-R(T)]\right\},
\end{aligned}
$$


and define

$$
(\Phi x)(t)= \begin{cases}(\Phi x)(T), & t \in\left[t_{0}, T\right] \\ -p(t) x(\tau(t))+\int_{T}^{t} \frac{1}{r(\eta)}\left[\frac{\varepsilon}{3}+\int_{\eta}^{\infty} q(\zeta) G(x(\sigma(\zeta))) d \zeta\right] d \eta, & t \geq T .\end{cases}
$$

For every $x \in M$,

$$
\begin{aligned}
(\Phi x)(t) & \geq \int_{T}^{t} \frac{1}{r(\eta)}\left[\frac{\varepsilon}{3}+\int_{\eta}^{\infty} q(\zeta) G(x(\sigma(\zeta))) d \zeta\right] d \eta \\
& \geq \frac{\varepsilon}{3} \int_{T}^{t} \frac{d \eta}{r(\eta)}=\frac{\varepsilon}{3}[R(t)-R(T)]
\end{aligned}
$$

and $x(t) \leq \varepsilon R(t)$ implies that

$$
\begin{aligned}
(\Phi x)(t) & \leq-p(t) x(\tau(t))+\frac{2 \varepsilon}{3} \int_{T}^{t} \frac{d \eta}{r(\eta)} \\
& \leq a \varepsilon[R(\tau(t))-R(T)]+\frac{2 \varepsilon}{3}[R(t)-R(T)] \\
& \leq a \varepsilon[R(t)-R(T)]+\frac{2 \varepsilon}{3}[R(t)-R(T)] \\
& =\left(a+\frac{2}{3}\right) \varepsilon[R(t)-R(T)] \\
& \leq \varepsilon[R(t)-R(T)]
\end{aligned}
$$

implies that $(\Phi x)(t) \in M$. Define $u_{n}:\left[t_{0},+\infty\right) \rightarrow \mathbb{R}$ by the recursive formula

$$
u_{n}(t)=\left(\Phi u_{n-1}\right)(t), \quad n \geq 1,
$$

with the initial condition

$$
u_{0}(t)= \begin{cases}0, & t \in\left[t_{0}, T\right] \\ \frac{\varepsilon}{3}[R(t)-R(T)], & t \geq T\end{cases}
$$

Inductively it is easy to verify that

$$
\frac{\varepsilon}{3}[R(t)-R(T)] \leq u_{n-1}(t) \leq u_{n}(t) \leq \varepsilon[R(t)-R(T)],
$$

for $t \geq T$. Therefore, for $t \geq t_{0}, \lim _{n \rightarrow \infty} u_{n}(t)$ exists. By the Lebesgue's dominated convergence theorem, $u \in M$ and $(\Phi u)(t)=u(t)$, where $u(t)$ is a solution of (1.1) such that $u(t)>0$. Hence, $(A 5)$ is necessary. This completes the proof of the theorem.

Theorem 2.2. Assume that (A1)-(A3) hold and $-1<-a \leq p(t) \leq 0, a>0$ for $t \in \mathbb{R}_{+}$. Then every unbounded solution of (1.1) oscillates if and only if (A5) holds for every $\varepsilon>0$.

Proof. Without loss of generality, suppose the contrary that $x$ is an eventually positive unbounded solution of (1.1). Then, there exists $t_{1} \geq t_{0}$ such that $x(t)>0, x(\tau(t))>0$ and $x(\sigma(t))>0$ for $t \geq t_{1}$. Proceeding as in the proof of Lemma 2.1, we see $r z^{\prime}$ is 
nonincreasing and $z$ is monotonic on $\left[t_{2}, \infty\right)$, where $t_{2} \geq t_{1}$. We have the following two possible cases.

Case 1. Let $z(t)<0$ for $t \geq t_{2}$. The case is same as in proof of Theorem 2.1.

Case 2. Let $z(t)>0$ for $t \geq t_{2}$. By Lemma 2.1, (2.1) holds for $t \geq t_{3}$. Since $z(t)$ is unbounded and monotonic increasing, then it follows that

$$
\lim _{t \rightarrow \infty} \frac{z(t)}{R(t)}=\lim _{t \rightarrow \infty} \frac{z^{\prime}(t)}{R^{\prime}(t)}=\lim _{t \rightarrow \infty} r(t) z^{\prime}(t)=\alpha<\infty .
$$

If $\alpha=0$, then $\lim _{t \rightarrow \infty} R(t)=+\infty$ implies that $\lim _{t \rightarrow \infty} z(t)<+\infty$, which is absurd (because of unbounded $z(t)$ ). Hence $\alpha \neq 0$. Therefore, there exists a constant $\varepsilon>0$ and a $t_{2}>t_{1}$ such that $z(t) \geq \varepsilon R(t)$ for $t \geq t_{2}$. Consequently, $x(t) \geq z(t) \geq \varepsilon R(t)$ for $t \geq t_{2}$. Using $x(t) \geq \varepsilon R(t)$ in (2.2) and then integrating from $t_{2}$ to $+\infty$, we obtain a contradiction to $(A 5)$ for every $\varepsilon>0$.

The case where $x$ is eventually negative unbounded solution is very similar and we omit it here.

The necessary part is same as in Theorem 2.1. This completes the proof of the theorem.

Theorem 2.3. Assume that $(A 1)-(A 4)$ hold and $-1<-a \leq p(t) \leq 0$, where $a>0$, $t \in \mathbb{R}_{+}$. Then every solution of (1.1) oscillates or converges to zero if and only if (A5) holds for every $\varepsilon>0$.

Proof. Without loss of generality, suppose the contrary that $x$ is an eventually positive solution of (1.1). Then, there exists $t_{1} \geq t_{0}$ such that $x(t)>0, x(\tau(t))>0$ and $x(\sigma(t))>0$ for $t \geq t_{1}$. Proceeding as in the proof of Lemma 2.1, we see $r z^{\prime}$ is nonincreasing and, $r z^{\prime}$ and $z$ is monotonic on $\left[t_{2}, \infty\right)$, where $t_{2} \geq t_{1}$. By Lemma 2.1, we have the following three possible cases.

Case 1. Let $z(t)<0, r(t) z^{\prime}(t)<0$ for $t \geq t_{2}$. Since $z(t)<0$ implies $z(t)$ is bounded due to Theorem 2.1 and $r(t) z^{\prime}(t)<0$ implies that $z(t)$ is unbounded due to Lemma 2.1, a contradiction.

Case 2. Assume that $z(t)<0, r(t) z^{\prime}(t)>0$ holds for $t \geq t_{2}$. Therefore,

$$
\begin{aligned}
0 \geq \lim _{t \rightarrow \infty} z(t) & =\limsup _{t \rightarrow \infty} z(t) \\
& \geq \limsup _{t \rightarrow \infty}(x(t)-a x(\tau(t))) \\
& \geq \limsup _{t \rightarrow \infty} x(t)+\liminf _{t \rightarrow \infty}(-a x(\tau(t))) \\
& =(1-a) \limsup _{t \rightarrow \infty} x(t),
\end{aligned}
$$

implies that $\limsup _{t \rightarrow \infty} x(t)=0$ and hence $\lim _{t \rightarrow \infty} x(t)=0$.

Case 3. Let $z(t)>0, r(t) z^{\prime}(t)>0$ for $t \geq t_{2}$. The case follows from Theorem 2.1.

Hence, $(A 5)$ is a sufficient condition. The case where $x$ is negative solution is similar and we omit it here. 
The necessary part is same as in the Theorem 2.1. Thus, the proof of the theorem is complete.

Theorem 2.4. Assume that (A1)-(A3) hold and $-1<-a \leq p(t) \leq 0$ such that $r(t) \geq r(\sigma(t))$ for $a>0, t \in \mathbb{R}_{+}$. Furthermore, assume that

(A6) $G$ is strictly superlinear, that is, $\frac{G(u)}{u^{\beta}} \geq \frac{G(v)}{v^{\beta}}, u \geq v>0, \beta>1$,

holds. Then every solution of (1.1) either oscillates or converges to zero if and only if

(A7) $\int_{0}^{\infty} \frac{1}{r(\eta)}\left[\int_{\eta}^{\infty} q(\zeta) d \zeta\right] d \eta=+\infty$.

Proof. For the sufficient part, we use the same type of argument as in the proof of Theorem 2.3 for first two cases of the pair $z(t)$ and $r(t) z^{\prime}(t)$. Let us consider the Case 3 for $t \geq t_{1}$. By Remark 2.1, there exists a constant $\varepsilon>0$ and $t_{2}>t_{1}$ such that $z(\sigma(t)) \geq \varepsilon$ for $t \geq t_{2}$. Consequently,

$$
\begin{aligned}
G(z(\sigma(t))) & =\frac{G(z(\sigma(t)))}{z^{\beta}(\sigma(t))} z^{\beta}(\sigma(t)) \\
& \geq \frac{G(\varepsilon)}{\varepsilon^{\beta}} z^{\beta}(\sigma(t)),
\end{aligned}
$$

for $t \geq t_{2}$. Therefore, (2.4) becomes

$$
\begin{aligned}
r(t) z^{\prime}(t) & \geq \frac{G(\varepsilon)}{\varepsilon^{\beta}} \int_{t}^{\infty} q(\eta) z^{\beta}(\sigma(\eta)) d \eta, \\
& \geq \frac{G(\varepsilon)}{\varepsilon^{\beta}}\left[\int_{t}^{\infty} q(\eta) d \eta\right] z^{\beta}(\sigma(t)),
\end{aligned}
$$

that is,

$$
r(\sigma(t)) z^{\prime}(\sigma(t)) \geq \frac{G(\varepsilon)}{\varepsilon^{\beta}}\left[\int_{t}^{\infty} q(\eta) d \eta\right] z^{\beta}(\sigma(t)),
$$

for $t \geq t_{2}$, implies that

$$
\begin{aligned}
z^{\prime}(\sigma(t)) & \geq \frac{G(\varepsilon)}{\varepsilon^{\beta} r(\sigma(t))}\left[\int_{t}^{\infty} q(\eta) d \eta\right] z^{\beta}(\sigma(t)) \\
& \geq \frac{G(\varepsilon)}{\varepsilon^{\beta}} \frac{z^{\beta}(\sigma(t))}{r(t)}\left[\int_{t}^{\infty} q(\eta) d \eta\right] .
\end{aligned}
$$

Integrating the last inequality from $t_{2}$ to $+\infty$, we get

$$
\frac{G(\varepsilon)}{\varepsilon^{\beta}} \int_{t_{2}}^{\infty} \frac{1}{r(\eta)}\left[\int_{\eta}^{\infty} q(\zeta) d \zeta\right] d \eta \leq \int_{t_{2}}^{\infty} \frac{z^{\prime}(\sigma(\eta))}{z^{\beta}(\sigma(\eta))} d \eta<\infty,
$$

which is a contradiction to $(A 7)$.

The case where $x$ is eventually negative solution is omitted since it can be dealt similarly. 
Next, we show that $(A 7)$ is necessary. Assume that $(A 7)$ fails to hold and let

$$
G(\varepsilon) \int_{T}^{t} \frac{1}{r(\eta)}\left[\int_{\eta}^{\infty} q(\zeta) d \zeta\right] d \eta \leq \frac{\varepsilon}{3}, \quad T \geq T^{*}
$$

where $\varepsilon>0$ is a constant. Consider

$$
M=\left\{x \in C\left(\left[t_{0}, \infty\right), \mathbb{R}\right): x(t)=\frac{\varepsilon}{3}, t \in\left[t_{0}, T\right], \frac{\varepsilon}{3} \leq x(t) \leq \varepsilon, \text { for } t \geq T\right\},
$$

and define

$$
(\Phi x)(t)= \begin{cases}\frac{\varepsilon}{3}, & t \in\left[t_{0}, T\right], \\ -p(t) x(\tau(t))+\frac{\varepsilon}{3}+\int_{T}^{t} \frac{1}{r(\eta)}\left[\int_{\eta}^{\infty} q(\zeta) G(x(\sigma(\zeta))) d \zeta\right] d \eta, & t \geq T,\end{cases}
$$

for every $x \in M,(\Phi x)(t) \geq \frac{\varepsilon}{3}$ and

$$
\begin{aligned}
(\Phi x)(t) & \leq a \varepsilon+\frac{\varepsilon}{3}+G(\varepsilon) \int_{T}^{t} \frac{1}{r(\eta)}\left[\int_{\eta}^{\infty} q(\zeta) d \zeta\right] d \eta \\
& \leq a \varepsilon+\frac{\varepsilon}{3}+\frac{\varepsilon}{3}=\left(a+\frac{2}{3}\right) \varepsilon \\
& \leq \varepsilon
\end{aligned}
$$

implies that $\Phi x \in M$. The rest of the proof follows from Theorem 2.1. This completes the proof of the theorem.

Theorem 2.5. Assume that (A1)-(A3), (A6) hold and $0 \leq p(t) \leq a<1$ such that $r(t) \geq r(\sigma(t))$ for $t \in \mathbb{R}_{+}$. Furthermore, assume that $G$ is Lipschitzian on the interval of the form $[c, d], 0<c<d<\infty$. Then every solution of (1.1) oscillates if and only if $(A 7)$ holds.

Proof. Suppose the contrary that $x$ is a nonoscillatory solution of (1.1). Then, there exists $t_{1} \geq t_{0}$ such that either $x(t)>0$ or $x(t)<0$ for $t \geq t_{1}$. Assume that $x(t)>0$, $x(\tau(t))>0$ and $x(\sigma(t))>0$ for $t \geq t_{1}$. Clearly, $z$ defined by (2.1) is positive on $\left[t_{1}, \infty\right)$. By Lemma 2.1 and Remark 2.1, there exists $\varepsilon>0$ such that $z(t) \geq \varepsilon$ for $t \geq t_{2}$, where $t_{2} \geq t_{1}$. On the other hand, $z$ being increasing implies that

$$
\begin{aligned}
(1-a) z(t) & \leq(1-p(t)) z(t) \leq z(t)-p(t) z(\tau(t)) \\
& =x(t)-p(t) p(\tau(t)) x(\tau(\tau(t))) \leq x(t),
\end{aligned}
$$

for $t \geq t_{3}$, where $t_{3} \geq t_{2}$. Consequently, (1.1) becomes

$$
\left(r(t) z^{\prime}(t)\right)^{\prime}+q(t) G((1-a) z(\sigma(t))) \leq 0,
$$


for $t \geq t_{3}$. Using $(A 6)$ it follows that

$$
\begin{aligned}
G((1-a) z(\sigma(t))) & =\frac{G((1-a) z(\sigma(t)))}{(1-a)^{\beta} z^{\beta}(\sigma(t))}(1-a)^{\beta} z^{\beta}(\sigma(t)) \\
& \geq \frac{G(\varepsilon(1-a))}{\varepsilon^{\beta}(1-a)^{\beta}}(1-a)^{\beta} z^{\beta}(\sigma(t)) .
\end{aligned}
$$

The remaining portion of the sufficient part follows from Theorem 2.4.

Conversely, suppose that $(A 7)$ fails to hold. Then there exists $T \geq T^{*}$ such that

$$
\int_{T}^{\infty} \frac{1}{r(\eta)}\left[\int_{\eta}^{\infty} q(\zeta) d \zeta\right] d \eta<\frac{1-a}{5 K}
$$

where $K=\max \left\{K_{1}, G(1)\right\}$ and $K_{1}$ is the Lipschitz constant of $G$ on $\left[\frac{7(1-a)}{10}, 1\right]$ for $t \geq t_{0}$. Let $X=B C\left(\left[t_{0}, \infty\right), \mathbb{R}\right)$ be the space of real valued continuous functions on $\left[t_{0}, \infty\right)$. Indeed, $X$ is a Banach space with respect to sup norm defined by

$$
\|x\|=\sup \left\{|x(t)|: t \geq t_{0}\right\} .
$$

Define

$$
S=\left\{u \in X: \frac{7(1-a)}{10} \leq u(t) \leq 1, t \geq t_{0}\right\} .
$$

We notice that $S$ is a closed convex subspace of $X$. Let $\Phi: S \rightarrow S$ be such that

$$
(\Phi x)(t)= \begin{cases}(\Phi x)(T), & t \in\left[t_{0}, T\right] \\ -p(t) x(\tau(t))+\frac{9+a}{10}-\int_{t}^{\infty} \frac{1}{r(\eta)}\left[\int_{\eta}^{\infty} q(\zeta) G(x(\sigma(\zeta))) d \zeta\right] d \eta, & t \geq T .\end{cases}
$$

For every $x \in X,(\Phi x)(t) \leq \frac{9+a}{10} \leq 1$ and

$$
(\Phi x)(t) \geq-a+\frac{9+a}{10}-\frac{1-a}{5}=\frac{7}{10}(1-a),
$$

implies that $\Phi(x) \in S$. Now for $x_{1}, x_{2} \in S$, we have

$$
\begin{aligned}
\left|\left(\Phi x_{1}\right)(t)-\left(\Phi x_{2}\right)(t)\right| \leq & a\left|x_{1}(\tau(t))-x_{2}(\tau(t))\right| \\
& +\int_{t}^{\infty} \frac{1}{r(\eta)}\left[\int_{\eta}^{\infty} q(\zeta)\left|G\left(x_{1}(\sigma(\zeta))\right)-G\left(x_{2}(\sigma(\zeta))\right)\right| d \zeta\right] d \eta
\end{aligned}
$$

that is,

$$
\begin{aligned}
\left|\left(\Phi x_{1}\right)(t)-\left(\Phi x_{2}\right)(t)\right| & \leq a\left\|x_{1}-x_{2}\right\|+\left\|x_{1}-x_{2}\right\| K_{1} \int_{t}^{\infty} \frac{1}{r(\eta)}\left[\int_{\eta}^{\infty} q(\zeta) d \zeta\right] d \eta \\
& \leq\left(a+\frac{1-a}{5}\right)\left\|x_{1}-x_{2}\right\| \\
& =\frac{1+4 a}{5}\left\|x_{1}-x_{2}\right\| .
\end{aligned}
$$


Therefore, $\left\|\Phi x_{1}-\Phi x_{2}\right\| \leq \frac{1+4 a}{5}\left\|x_{1}-x_{2}\right\|$ implies that $\Phi$ is a contraction. By using Banach's contraction mapping principle, it follows that $\Phi$ has a unique fixed point $x(t)$ in $\left[\frac{7(1-a)}{10}, 1\right]$. Hence, $(A 7)$ is the necessary condition for oscillation of (1.1). This completes the proof of the theorem.

Theorem 2.6. Assume that (A1)-(A3) hold and $0 \leq p(t) \leq a<1$ for $t \in \mathbb{R}_{+}$. Furthermore, assume that $G$ be Lipschitzian on intervals of the form $[c, d], 0<c<$ $d<\infty$. Then every bounded solutions of (1.1) oscillates if and only if (A7) holds.

Proof. Proceeding as in proof of the Theorem 2.5 we have obtained $x(t) \geq(1-a) z(t) \geq$ $(1-a) \varepsilon=\varepsilon_{1}$. Consequently, (1.1) becomes

$$
\left(r(t) z^{\prime}(t)\right)^{\prime}+q(t) G\left(\varepsilon_{1}\right) \leq 0 .
$$

Twice integration on last inequality yields a contradiction to $(A 7)$. The necessary part is same as in the proof of Theorem 2.5. Hence the details are omitted. Thus the proof of theorem is complete.

Theorem 2.7. Assume that (A1)-(A3) hold and $-\infty<-a_{1} \leq p(t) \leq-a_{2}<-1$ such that $3 a_{2}>a_{1}$ for $t \in \mathbb{R}_{+}$where $a_{1}, a_{2}>0$. Let $G$ be Lipschitzian on intervals of the form $[c, d], 0<c<d<\infty$. Then every bounded solution of (1.1) oscillates or tends to zero if and only if $(A 7)$ holds.

Proof. Without loss of generality, suppose the contrary that $x$ is an eventually positive solution of (1.1). Then, there exists $t_{1} \geq t_{0}$ such that $x(t)>0, x(\tau(t))>0$ and $x(\sigma(t))>0$ for $t \geq t_{1}$. Proceeding as in the proof of Lemma 2.1, we see $r z^{\prime}$ is nonincreasing and, $r z^{\prime}$ and $z$ is monotonic on $\left[t_{2}, \infty\right)$, where $t_{2} \geq t_{1}$. Since $x(t)$ is bounded, then by (1.4), $z(t)$ is bounded and hence $\lim _{t \rightarrow \infty} z(t)$ exists. It is easy to see that the case $z(t)<0, r(t) z^{\prime}(t)<0$ is not possible. Using the proof of Lemma 2.2 , we conclude that the case $z(t)>0, r(t) z^{\prime}(t)<0$ does not arise. Therefore, we have following two cases.

Case 1. Let $z(t)>0, r(t) z^{\prime}(t)>0$ for $\left[t_{3}, \infty\right), t_{3}>t_{2}$. Then we can find a constant $\varepsilon>0$ and $t_{4}>t_{3}$ such that $z(\sigma(t)) \geq \varepsilon$ for $t \geq t_{4}$, that is, $x(\sigma(t)) \geq z(\sigma(t)) \geq \varepsilon$ for $t \geq t_{4}$. Hence, (1.1) becomes

$$
\left(r(t) z^{\prime}(t)\right)^{\prime}+G(\varepsilon) q(t) \leq 0, t \geq t_{4} .
$$

Twice integration on last inequality gives a contradiction to $(A 7)$.

Case 2. Let $z(t)<0, r(t) z^{\prime}(t)>0$ for $\left[t_{3}, \infty\right), t_{3}>t_{2}$. We claim that $\lim _{t \rightarrow \infty} z(t)=$ 0 . If not, there exist $\alpha<0$ and $t_{4}>t_{3}$ such that $z\left(\tau^{-1}(\sigma(t))\right)<\alpha$ for $t \geq t_{4}$. Hence, $z(t) \geq-a_{1} x(\tau(t))$ implies that $x(t) \geq-a_{1}^{-1} z\left(\tau^{-1}(t)\right)$, that is, $x(\sigma(t)) \geq$ $-a_{1}^{-1} z\left(\tau^{-1}(\sigma(t))\right) \geq-a_{1}^{-1} \alpha$ for $t \geq t_{4}$. Consequently, (1.1) reduces to

$$
\left(r(t) z^{\prime}(t)\right)^{\prime}+G\left(-a_{1}^{-1} \alpha\right) q(t) \leq 0
$$


for $t \geq t_{4}$. Using the same type of argument as in the former case, we get a contradiction to $(A 7)$. Thus, our claim holds and hence

$$
\begin{aligned}
0=\lim _{t \rightarrow \infty} z(t) & =\liminf _{t \rightarrow \infty}(x(t)+p(t) x(\tau(t))) \\
& \leq \liminf _{t \rightarrow \infty}\left(x(t)-a_{2} x(\tau(t))\right) \\
& \leq \limsup _{t \rightarrow \infty} x(t)+\liminf _{t \rightarrow \infty}\left(-a_{2} x(\tau(t))\right) \\
& =\left(1-a_{2}\right) \limsup _{t \rightarrow \infty} x(t),
\end{aligned}
$$

implies that $\lim \sup _{t \rightarrow \infty} x(t)=0\left[\because 1-a_{2}<0\right]$. Therefore, $\lim _{t \rightarrow \infty} x(t)=0$.

The case where $x$ is negative bounded solution is very similar and we omit it here.

For the necessary part, it is possible to find $T \geq T^{*}$ such that

$$
\int_{T}^{\infty} \frac{1}{r(\eta)}\left[\int_{\eta}^{\infty} q(\zeta) d \zeta\right] d \eta<\frac{a_{2}-1}{3 K}
$$

where $K=\max \left\{K_{1}, G(1)\right\}$ and $K_{1}$ is the Lipschitz constants of $G$ on $[a, 1]$, where $a=\frac{\left(a_{2}-1\right)\left(3 a_{2}-a_{1}\right)}{3 a_{1} a_{2}}$. Let $X=B C\left(\left[t_{0}, \infty\right), \mathbb{R}\right)$ be the space of real valued continuous functions defined on $\left[t_{0}, \infty\right)$. Indeed, $X$ is a Banach space with the sup norm defined by

$$
\|x\|=\sup \left\{|x(t)|: t \geq t_{0}\right\}
$$

Define

$$
S=\left\{u \in X: a \leq u(t) \leq 1, t \geq t_{0}\right\}
$$

and we note that $S$ is a closed convex subspace of $X$. Let $\Phi: S \rightarrow S$ be such that

$$
(\Phi x)(t)= \begin{cases}(\Phi x)(T), & t \in\left[t_{0}, T\right] \\ -\frac{x\left(\tau^{-1}(t)\right)}{p\left(\tau^{-1}(t)\right)}-\frac{a_{2}-1}{p\left(\tau^{-1}(t)\right)} & \\ +\frac{1}{p\left(\tau^{-1}(t)\right)} \int_{T}^{\tau^{-1}(t)} \frac{1}{r(\eta)}\left[\int_{\eta}^{\infty} q(\zeta) G(x(\sigma(\zeta))) d \zeta\right] d \eta, & t \geq T .\end{cases}
$$

For every $x \in S$,

$$
(\Phi x)(t) \leq-\frac{x\left(\tau^{-1}(t)\right)}{p\left(\tau^{-1}(t)\right)}-\frac{a_{2}-1}{p\left(\tau^{-1}(t)\right)} \leq \frac{1}{a_{2}}+\frac{a_{2}-1}{a_{2}}=1
$$


and

$$
\begin{aligned}
(\Phi x)(t) & \geq-\frac{a_{2}-1}{p\left(\tau^{-1}(t)\right)}+\frac{1}{p\left(\tau^{-1}(t)\right)} \int_{T}^{\tau^{-1}(t)} \frac{1}{r(\eta)}\left[\int_{\eta}^{\infty} q(\zeta) G(x(\sigma(\zeta))) d \zeta\right] d \eta \\
& \geq-\frac{a_{2}-1}{a_{1}}+\frac{G(1)}{p\left(\tau^{-1}(t)\right)} \int_{T}^{\tau^{-1}(t)} \frac{1}{r(\eta)}\left[\int_{\eta}^{\infty} q(\zeta) d \zeta\right] d \eta \\
& \geq-\frac{a_{2}-1}{a_{1}}-\frac{G(1)}{a_{2}} \int_{T}^{\infty} \frac{1}{r(\eta)}\left[\int_{\eta}^{\infty} q(\zeta) d \zeta\right] d \eta \\
& \geq-\frac{a_{2}-1}{a_{1}}-\frac{a_{2}-1}{3 a_{2}}=a,
\end{aligned}
$$

implies that $\Phi x \in S$. Now for $x_{1}, x_{2} \in S$, we have

$$
\begin{aligned}
\left|\left(\Phi x_{1}\right)(t)-\left(\Phi x_{2}\right)(t)\right| \leq & \frac{1}{\left|p\left(\tau^{-1}(t)\right)\right|}\left|x_{1}\left(\tau^{-1}(t)\right)-x_{2}\left(\tau^{-1}(t)\right)\right|+\frac{K_{1}}{\left|p\left(\tau^{-1}(t)\right)\right|} \\
& \times \int_{T}^{\tau^{-1}(t)} \frac{1}{r(\eta)}\left[\int_{\eta}^{\infty}\left|x_{1}(\sigma(\zeta))-x_{2}(\sigma(\zeta))\right| q(\zeta) d \zeta\right] d \eta \\
\leq & \frac{1}{a_{2}}\left\|x_{1}-x_{2}\right\|+\frac{a_{2}-1}{3 a_{2}}\left\|x_{1}-x_{2}\right\| \\
= & \gamma\left\|x_{1}-x_{2}\right\|,
\end{aligned}
$$

implies that

$$
\left\|\Phi x_{1}-\Phi x_{2}\right\| \leq \gamma\left\|x_{1}-x_{2}\right\|,
$$

where $\gamma=\frac{1}{a_{2}}\left(1+\frac{a_{2}-1}{3}\right)<1$. Therefore, $\Phi$ is a contraction. Hence by the Banach's contraction mapping principle $\Phi$ has a unique fixed point $x \in S$. It is easy to see that $\lim _{t \rightarrow \infty} x(t) \neq 0$. This completes the proof of the theorem.

\section{Discussion And ExAmple}

It is worth observation that we could succeed partially to establish the oscillation of all solutions of the nonlinear equation (1.1), when $|p(t)|<\infty$. We failed to obtain the necessary and sufficient conditions in the range $1 \leq p(t)<\infty$ and $p(t) \equiv-1$. Therefore, the undertaken problem is incomplete for all range of $p(t)$.

Remark 3.1. In Theorems 2.2, 2.6 and 2.7, $G$ could be linear, sublinear or superlinear.

We conclude this section with the following examples to illustrate our main results: Example 3.1. Consider the delay differential equations

$$
\frac{d}{d t}\left[t \frac{d}{d t}\left[x(t)-3 x\left(e^{-\pi} t\right)\right]\right]+\frac{4}{t} x(t)=0, \quad \text { for } t \geq 1,
$$

where $r(t):=t, p(t): \equiv-3, \tau(t):=e^{-\pi} t, q(t):=\frac{4}{t^{2}}, \sigma(t):=t$ and $G(u):=u$ for $t \geq 1$ and $u \in \mathbb{R}$. It can be easily shown that Theorem 2.7 applies to (3.1). Thus, 
every bounded solution oscillates or converges to zero asymptotically. Obviously, $x(t)=\sin \left(\ln \left(t^{2}\right)\right)$ for $t \geq 1$ is an oscillating solution.

Acknowledgements. The author is thankful to the referees for their suggestions and necessary corrections in the completion of this paper.

This work was supported by the Department of Science and Technology (DST), New Delhi, India, through the letter No. DST/INSPIRE Fellowship/2014/140 (dated Sept. 15, 2014).

This work was accepted when the author visited at the University of Exeter, UK under the Newton-Bhabha Ph.D. placement programme funded by DST (India Govt.) and British Council (British Govt.) through the letter No. DST/INSPIRE/NBHF/ 2017/5 (dated Feb. 13, 2018).

\section{REFERENCES}

[1] R. P. Agarwal, M. Bohner, T. Li and C. Zhang, Oscillation of second order differential equations with a sublinear neutral term, Carpathian J. Math. 30 (2014), 1-6.

[2] A. Ardjouni and A. Djoudi, Periodic solutions for impulsive neutral dynamic equations with infinite delay on time scales, Kragujevac J. Math. 42(1) (2018), 69-82.

[3] B. Baculikova and J. Dzurina, Oscillation theorems for second order neutral differential equations, Comput. Math. Appl. 61 (2011), 94-99.

[4] B. Baculikova and J. Dzurina, Oscillation theorems for second order nonlinear neutral differential equations, Comput. Math. Appl. 62 (2011), 4472-4478.

[5] B. Baculikova, T. Li and J. Dzurina, Oscillation theorems for second order neutral differential equations, Electron. J. Qual. Theory Differ. Equ. 74 (2011), 1-13.

[6] J. Džurina, Oscillation theorems for second order advanced neutral differential equations, Tatra Mt. Math. Publ. 48 (2011), 61-71.

[7] T. H. Hildebrandt, Introduction to the Theory of Integration, Pure and Applied Mathematics 13, Academic Press, New York, London, 1963.

[8] J. Hale, Theory of Functional Differential Equations, Applied Mathematical Sciences 3, 2nd ed. Springer-Verlag, New York, Heidelberg, Berlin, 1977.

[9] B. Karpuz and S. S. Santra, Oscillation theorems for second-order nonlinear delay differential equations of neutral type, Hacet. J. Math. Stat. DOI 10.15672/HJMS.2017.542.

[10] T. Li and Y. V. Rogovchenko, Oscillation theorems for second order nonlinear neutral delay differential eqquations, Abstr. Appl. Anal. 2014 (2014), Paper ID 594190, 1-5.

[11] M. Mikic, Note about asymptotic behaviour of positive solutions of superlinear differential equation of emden-fowler type at zero, Kragujevac J. Math. 40(1) (2016), 105-112.

[12] S. Pinelas and S. S. Santra, Necessary and sufficient condition for oscillation of nonlinear neutral first-order differential equations with several delays, J. Fixed Point Theory Appl. 20(1) (2018), $1-13$.

[13] Y. Qian and R. Xu, Some new osciilation criteria for higher order quasi-linear neutral delay differential equations, Differ. Equ. Appl. 3 (2011), 323-335.

[14] S. S. Santra, Oscillation criteria for nonlinear neutral differential equations of first order with several delays, Mathematica 57(80)(1-2) (2015), 75-89.

[15] S. S. Santra, Necessary and sufficient condition for oscillation of nonlinear neutral first order differential equations with several delays, Mathematica 58(81)(1-2) (2016), 85-94.

[16] S. S. Santra, Existence of positive solution and new oscillation criteria for nonlinear first order neutral delay differential equations, Differ. Equ. Appl. 8(1) (2016), 33-51. 
[17] S. S. Santra, Oscillation analysis for nonlinear neutral differential equations of second order with several delays, Mathematica 59(82)(1-2) (2017), 111-123.

[18] A. K. Tripathy, B. Panda and A. K. Sethi, On oscillatory nonlinear second order neutral delay differential equations, Differ. Equ. Appl. 8 (2016), 247-258.

[19] Q. Yang and Z. Xu, Oscillation criteria for second order quasi-linear neutral delay differential equations on time scales, Comput. Math. Appl. 62 (2011), 3682-3691.

[20] L. Ye and Z. Xu, Oscillation criteria for second order quasilinear neutral delay differential equations, Appl. Math. Comput. 207 (2009), 388-396.

${ }^{1}$ Department of MAThematics,

SAMBALPUR UNIVERSITY,

SAMBALPUR 768019, INDIA

${ }^{2}$ Department of MAThematics,

UNIVERSITY OF EXETER,

EXETER EX4 4QF, UK

Email address: shyam01.math@gmail.com

Email address: shyam01.math@suniv.ac.in

Email address: sss215@exeter.ac.uk 